\title{
High repetition rate seeded free electron laser with an optical klystron in high-gain harmonic generation
}

\author{
Georgia Paraskaki®, ${ }^{*}$ Enrico Allaria๑, and Evgeny Schneidmiller@ \\ Deutsches Elektronen-Synchrotron DESY, Notkestraße 85, 22607 Hamburg, Germany \\ Wolfgang Hillert $\odot$ \\ University of Hamburg, Luruper Chaussee 149, 22761 Hamburg, Germany
}

(Received 26 August 2021; accepted 23 November 2021; published 14 December 2021)

\begin{abstract}
Many high-gain free electron lasers worldwide are planning to incorporate seeding setups into their day-to-day operation. These techniques provide both longitudinal and transverse coherence and extended control of the output free electron laser radiation spectral properties. However, the output wavelength and repetition rate strongly depend on the properties of the seed laser system. With the laser peak power required for successful seeded operation, it is currently not possible to increase their repetition rate to an extent that it matches the electron bunch repetition rate of superconducting accelerators. Here, we investigate the advantages of a modification of standard seeding setups, by combining the seeding with the so-called optical klystron. With this new seeding setup, it is possible to decrease the seed laser power requirements and therefore, seed laser systems can increase their repetition rate at the same wavelength. We show simulation results in a high-gain harmonic generation (HGHG) setup for a range of harmonics (8th to 15th) and we verify the reduction of seed laser power required with an Optical Klystron HGHG scheme. Finally, we comment on the stability of the proposed setup to jitter sources and to shot-to-shot fluctuations and compare to the standard HGHG scheme.
\end{abstract}

DOI: 10.1103/PhysRevAccelBeams.24.120701

\section{INTRODUCTION}

High-gain free electron lasers (FELs) have been delivering light characterized by high brightness and wavelength tunability to user experiments for more than a decade $[1,2]$. At the same time, scientists push the limits in many directions to improve the available flux, the control, and the spectral quality of the output FEL radiation. An interesting milestone to be reached in the near future is to generate stable, fully coherent pulses at high-repetition rate. High repetition rate has been possible with superconducting linacs since 2005, when FLASH started operating at $1 \mathrm{MHz}$ in burst mode [3]. The European X-ray free electron laser (XFEL) is also a burst-mode FEL operating at 4.5 MHz since 2017 [4], while SHINE [5] and LCLS-II [6] aim to be the first continuous wave FELs at $1 \mathrm{MHz}$.

Even though there is currently no standard method to provide fully coherent pulses at these repetition rates, there has been ongoing research the last decades to increase the

\footnotetext{
*georgia.paraskaki@desy.de
}

Published by the American Physical Society under the terms of the Creative Commons Attribution 4.0 International license. Further distribution of this work must maintain attribution to the author(s) and the published article's title, journal citation, and DOI. repetition rate of fully coherent pulses with a particular interest in oscillator-based solutions. The oscillator can be used either as part of a multistage scheme that is combined with harmonic conversion [7-10] or as a direct source of radiation. The latter oscillator FELs are based on x-ray cavities which provide short wavelengths with full coherence and high repetition rate [11-16].

Self-amplified spontaneous emission (SASE) [17] is one of the standard methods in high-gain FELs to deliver light to experiments. It offers a wide range of wavelength tunability down to hard $\mathrm{x}$ rays [18-21] and with high repetition rate $[2,4]$, but it suffers from intrinsic limitations. The output longitudinal coherence of the FEL radiation is poor, as a result of the stochastic initiation of the FEL process, which also leads to shot-to-shot fluctuations of other output FEL pulse properties.

Techniques such as the self-seeding [22-24] and external seeding [25-27] are implemented to improve the longitudinal coherence. Self-seeding has been successfully demonstrated down to the hard x-ray regime with the generation of narrow and single line spectra. However, the shot-to-shot intensity stability still suffers from the SASE fluctuations, and the high repetition rate is challenging due to the heat load of the crystals used [28]. With external seeding techniques, like the echo-enabled harmonic generation (EEHG) $[27,29,30]$ and high-gain harmonic generation (HGHG) [25,31], an external seed laser source is 
used to prepare the electron bunches to emit coherently at a harmonic of the seed laser wavelength at the final amplification stage. These methods ensure both longitudinal coherence and shot-to-shot reproducibility and have allowed a great number of interesting experiments to become reality [32-34]. However, the dependence of the output FEL on the seed laser properties comes with a few downsides too, as it limits the shortest wavelength and the highest repetition rate possible.

The most common choices of seed laser systems for external seeding is in the ultraviolet (UV) wavelength range $[29,30,35]$. These systems offer sufficient pulse energies, wavelength and energy stability with fs duration, and wavelength tunability. Shorter wavelength seed laser systems, such as high-order harmonics generated in gas (HHG) [36], have been used in few direct seeding experiments $[37,38]$ and in harmonic conversion as well $[39,40]$, but have resulted to be unsuitable for user facilities due to the lack of peak power and flexibility. Currently, a conversion of up to two orders of magnitude in wavelength can be foreseen with the most efficient seeding method for this purpose, EEHG. Practically, this limits the shortest wavelength to the soft x-ray regime. At the same time, the UV seed lasers that are currently used for seeding are available at repetition rates of several tens of $\mathrm{Hz}$. The highly demanding parameters at higher repetition rates are not easily achievable, and one of the main limitations is the heat loading due to the peak powers they operate at. As a result, when electron bunches are available at $\mathrm{MHz}$ repetition rate, hundreds of thousands of electron bunches remain unused due to the lack of seed laser systems operating at the same repetition rate.

Here, we study in detail an alternative setup that can bridge this gap. This setup is based on the optical klystron (OK) scheme that was first introduced in Ref. [41], and was combined with external seeding only recently $[42,43]$. An OK can be used in combination with external seeding schemes to reduce the requirements in seed laser power. Our studies show that an optimized setup for this configuration can reduce the power requirements for the seed laser up to a factor of more than one thousand under certain conditions. At the same time, we show that even with a lowseed laser power it is possible to preserve the coherence of the output FEL on a shot-to-shot basis, thus significantly extending the previously reported results [42]. Since the heat load of laser systems is proportional to pulse energy and repetition rate, one can be traded for the other, allowing for higher repetition rate systems with current technology.

Additionally, by using shorter wavelength seed lasers that are available only at lower peak power, the OK-HGHG scheme allows an extension of the tuning range toward shorter wavelengths with respect to the single-stage HGHG scheme. Concerning the implementation of the OK-HGHG scheme, an advantage of this setup is that for facilities designed for EEHG, such as the new FLASH1 beamline proposed in the FLASH2020+ project [44], the existing setup is already sufficient and the OK-HGHG seeding can be implemented with no modifications. Finally, it was recently proposed that an OK-HGHG scheme can take advantage of the energy modulation induced in a dipole with the interaction of the laser beam and electron bunch [45]. A few additional considerations on increasing the repetition rate of seeded FELs can be found in Ref. [46] and an analytical study on several methods that have been proposed for high repetition rate seeded FEL can be found in Ref. [47].

In the following Sec. II, we introduce the OK-HGHG setup. To better understand the opportunities and limitations of the scheme, in Sec. III we simulate an OK-HGHG setup and we investigate the bunching mechanism and its stability to shot-to-shot jitter sources, in comparison with the standard HGHG seeding scheme. In Sec. IV, we select the 15th harmonic of a $300 \mathrm{~nm}$ seed laser wavelength and we optimize and present the output FEL. Finally, we discuss the impact of the lower signal to noise ratio on the shot-to-shot stability.

\section{MODIFYING AN HGHG SETUP TO INCLUDE AN OPTICAL KLYSTRON}

In a standard HGHG setup, an external seed laser source, a short undulator (modulator), a dispersive section, such as a chicane, and a longer undulator (amplifier) are required, as shown in Fig. 1. One seed laser pulse should be injected for each electron bunch, and together they interact along the modulator which is typically around two power gain lengths long. As a result of their interaction, the energy of the electron bunch is sinusoidally modulated in the longitudinal axis with a periodicity of a seed laser wavelength $\left(\lambda_{\text {seed }}\right)$. The chicane converts the energy modulation into a longitudinal density modulation. We quantify this density modulation with the so-called bunching factor $b_{\mathrm{h}}$ of a harmonic $h$, defined as

$$
b_{\mathrm{h}}=\left|J_{\mathrm{h}}(-h A B)\right| \exp \left(-\frac{1}{2} h^{2} B^{2}\right)
$$

where $A$ is the relative energy modulation, defined as the ratio of the amplitude of the energy modulation $\Delta E$ with respect to the uncorrelated energy spread $\sigma_{E}$, and $B$ is the relative dispersive strength of the chicane, $B=2 \pi R_{56} \sigma_{E} /$ $\left(\lambda_{\text {seed }} E\right)$, where $E$ is the electron beam mean energy and $R_{56}$

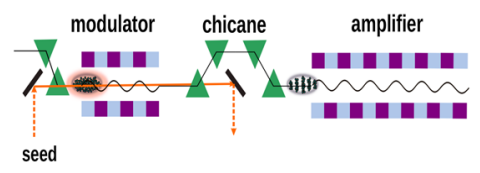

FIG. 1. In a standard HGHG setup, we need one modulator followed by a chicane, before the final FEL amplification at the amplifier. The amplifier is tuned to a harmonic of the seed laser wavelength. 
is the longitudinal dispersion of the chicane. Finally, $J_{\mathrm{h}}$ is the Bessel function of the first kind.

For a large $B$ the exponential decay of Eq. (1) minimizes the bunching. As a result, in order to obtain sufficient bunching at a harmonic of the seed laser, a large $A$ is required, and usually we take $A \simeq h$ for the harmonic number $h$. At the same time, the amplification process in the amplifier depends on the energy spread of the electron bunch upstream from the amplifier $\left(\sigma_{E}^{\prime}\right)$ relative to the electron beam energy and should not exceed the FEL parameter $\rho$ [17] upstream from the amplifier, which is typically in the order of $10^{-4}-10^{-3}$. For an energy-modulated electron beam, this means that $\left(\sqrt{\sigma_{E}^{2}+\Delta E^{2} / 2}\right) / E<\rho$. With a single-stage HGHG, we are typically limited to a highest harmonic of 15 , and this number strongly depends on the energy spread of the electron beam [48]. For harmonics up to the 15th, the amplitude of the energy modulation downstream from the modulator is expected to be roughly 5-15 times the uncorrelated energy spread. For typical EUV FEL parameters and since the energy modulation is proportional to the square root of the seed laser power $A \propto \sqrt{P_{\text {seed }}}$ [49], this means that the seed laser power at the modulator should be tens to hundreds of MW. When sufficient bunching is obtained in the order of a few percent, the individual electron microbunches emit radiation in sync at the amplifier downstream and fully coherent radiation at a harmonic of the seed laser wavelength is amplified.

We can reduce the seed laser power requirements with the addition of the OK, as shown in Fig. 2. Optical klystrons have been a useful tool for FELs since the 1970s [41], and have been widely used for gain control in oscillator FELs [50], for energy spread measurements, and to reduce the saturation length in SASE [51-53].

In the case of an OK-HGHG setup, as shown in Fig. 2, we need a seed laser source of a peak power that can induce a low-energy modulation of one to two times the uncorrelated energy spread after traversing the first modulator. Based on Ref. [53], if that is the case, then an optimization according to Eq. (3) is appropriate. This is normally not sufficient for seeding schemes and the generation of higher harmonics, but it is sufficient to increase the bunching at the fundamental wavelength with the right setting of the first

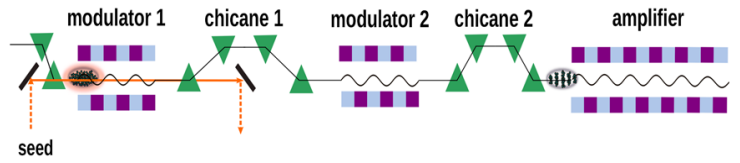

FIG. 2. In an OK-HGHG, we need two pairs of a modulator followed by a chicane. The two modulators are set to the same resonance, chicane 1 is responsible for increasing the bunching at the fundamental wavelength, while chicane 2 is responsible for longitudinal density modulation at a harmonic of the seed laser wavelength. The final FEL amplification of a harmonic of the seed laser wavelength is taking place at the amplifier. chicane $R_{56,1}$. This way, in modulator 2 we no longer need a seed laser, but the initial energy modulation is selfamplified further to an extent that it can be used to increase the bunching at a harmonic of the seed laser wavelength. The expected approximate gain in energy modulation with an optical klystron is [54]

$$
G_{A}=\frac{A_{2}}{A_{1}} \simeq \frac{I \times N}{\gamma \sigma_{\delta} I_{A}},
$$

where $A_{1}$ and $A_{2}$ are the energy modulation after the first and second modulator, respectively, $\sigma_{\delta}=\sigma_{E} / E$ where $\sigma_{E}$ is the uncorrelated energy spread, $I$ is the peak current, $\gamma$ is the electron beam energy, $N$ is the number of undulator periods of one segment, and $I_{A}=17 \mathrm{kA}$ is the Alfvén current. For this approximation, a thin beam and low gain in a single undulator are assumed and the effect of the emittance on the velocity spread is neglected.

In this setup, since the energy modulation is relatively low, the longitudinal dispersion of chicane 1 is optimized based on the electron beam energy spread as $[51,55,56]$

$$
R_{56,1}=\frac{\lambda_{\text {seed }}}{2 \pi \sigma_{\delta}} .
$$

The longitudinal dispersion of chicane 2 is optimized to increase the bunching at a harmonic of the seed laser wavelength based on Eq. (1).

\section{GAIN IN SEED LASER POWER WITH AN OPTICAL KLYSTRON}

\section{A. Comparing the performance of different setups}

In order to verify the benefits of the OK-HGHG, we first looked at the bunching mechanism with single-slice (timeindependent) simulations. We used the standard seeding scheme (see Fig. 1), and the one based on an optical klystron (see Fig. 2) to amplify the laser-induced modulation. For this study, we considered the electron beam, seed laser, and undulator parameters shown in Table I. These parameters are typical for EUV FELs and are also used as the basis for the future seeding plans at FLASH1 beamline in the FLASH2020+ upgrade [57]. All simulations shown in this paper were performed with the FEL simulation code GENESIS 1.3, version 4 [58].

The first comparison between the two methods is done by determining the minimum seed laser power required to achieve $8 \%$ bunching upstream from the amplifier for different harmonics. This amplitude of bunching is a good trade-off between a higher bunching that requires higher induced energy spread and a lower bunching that increases the saturation length and results in a lower signal to noise ratio. The seed laser wavelength is $300 \mathrm{~nm}$, and we studied the harmonics between the 8th and 15th, thus the output FEL wavelength is between $37.5 \mathrm{~nm}$ and $20 \mathrm{~nm}$. 
In order to optimize the standard HGHG scheme, we scan the seed laser power and the longitudinal dispersion $R_{56}$ of the chicane. Then, we select the combination of these two parameters with which we can obtain $8 \%$ bunching upstream from the amplifier, while the seed laser power (and thus the energy spread upstream from the amplifier $\sigma_{E}^{\prime}$ ) is minimum.

For the OK-HGHG, there are three available tuning parameters: the input seed laser power, the strength $R_{56,1}$ and $R_{56,2}$ of the first and second chicane, respectively (see Fig. 2). For low-seed laser power, the optimal setting of $R_{56,1}$ only depends on the electron beam slice energy spread and does not depend on the seed laser properties. Based on Eq. (3), it is set to $482 \mu \mathrm{m}$ and we keep it constant during our optimization for several harmonics, after verifying that this is the optimal longitudinal dispersion for several cases. Then, we optimize to obtain $8 \%$ bunching with minimal energy spread upstream from the amplifier for all harmonics. The remaining available tuning parameters for this purpose are the input seed laser power and the $R_{56,2}$ of the second chicane.

With the OK-HGHG, it is unavoidable to end up with a larger energy spread in order to achieve the same bunching compared to a standard HGHG scheme. This is because, with the OK-HGHG there are two different stages where we rotate the electron bunch distribution in the longitudinal phase space. Due to the intermediate gain, at the end of the second modulator the distribution is not perfectly sinusoidal on the longitudinal axis [Fig. 3(a)] as expected with the standard HGHG setup [Fig. 3(b)]. As a direct consequence, more energy modulation is required to achieve the same bunching. In an equivalent way, the same energy modulation yields smaller bunching for the OK-HGHG.

In Fig. 4, we present the results of this study. In Fig. 4a, we compare the required energy spread upstream from the amplifier $\left(\sigma_{\mathrm{E}}^{\prime}\right)$ to obtain $8 \%$ bunching by showing the ratio between the two schemes $\left(\sigma_{\mathrm{E}, \mathrm{HGHG}}^{\prime} / \sigma_{\mathrm{E}, \mathrm{OK}-\mathrm{HGHG}}^{\prime}\right)$. This way, we capture the anticipated increased energy spread that was demonstrated in Fig. 3. In Fig. 4(b), we show the ratio of the input seed laser power required with the standard HGHG scheme to the input seed laser power required with an OK-HGHG seeding scheme. In the nominal case, we used the parameters shown in Table I, and in all cases, the seed laser power required with the OK-HGHG was 500 to 300 times less than in a standard HGHG setup. The ratio is reduced for higher harmonics, however, even at the highest studied harmonic $(h=15)$, the gain in seed laser power is significant.

We additionally studied two different configurations to better understand the critical parameters for the OKHGHG. Initially, we studied the case where the electron beam peak current is increased by $50 \%$, from 500 to $750 \mathrm{~A}$. We assume that the energy spread is increased proportionally during the compression by $50 \%$ and is $112.5 \mathrm{keV}$. The new strength of the first chicane in the OK-HGHG is

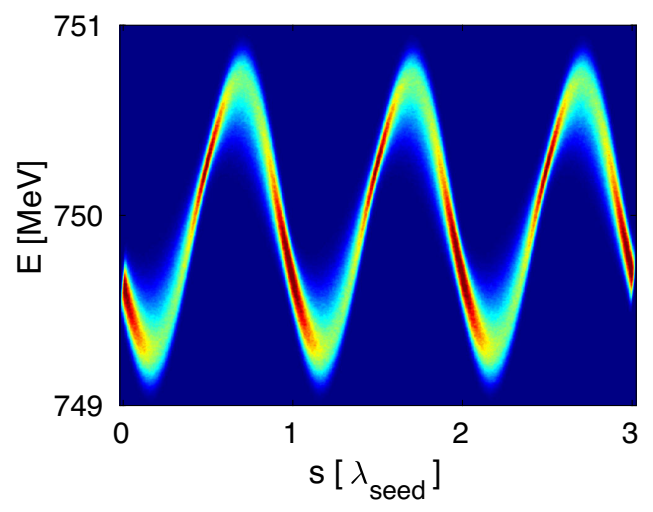

(a)

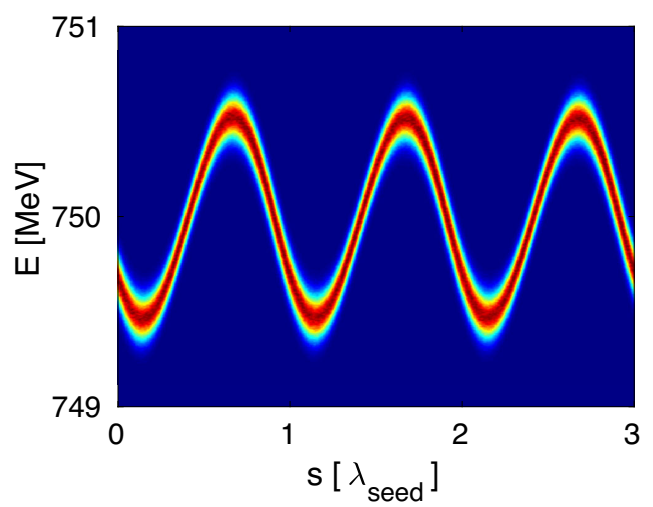

(b)

FIG. 3. (a) Longitudinal phase space after modulator 2 in an OK-HGHG setup (see Fig. 2). With an $R_{56,2}=51 \mu \mathrm{m}$, we get $b_{10}=8 \%$. (b) Longitudinal phase space after modulator in a standard HGHG setup (see Fig. 1). With an $R_{56}=67.25 \mu \mathrm{m}$, we get $b_{10}=8 \%$.

$R_{56,1}=318 \mu \mathrm{m}$ and it was optimized for the new energy spread value with Eq. (3). In both the OK-HGHG and the standard HGHG setup, the input seed laser power and the strength of the chicane upstream from the amplifier were optimized once more to obtain $8 \%$ bunching with the minimum possible seed laser power. The new power ratios are mildly increased, but overall there is no significant difference in the comparison of the two ratios. An increased peak current is directly associated with the decrease of one power gain length $\left(L_{\mathrm{g}}\right)$, in our case from 0.94 to $0.78 \mathrm{~m}$, thus the ratio of the gain lengths per modulator length ( $L_{\text {mod }}$ ) increases from $L_{\text {mod }} / L_{\mathrm{g}}=2.6$ to $L_{\text {mod }} / L_{\mathrm{g}}=3.1$. The increase of the energy spread requires larger energy modulation in both cases. The higher peak current does not significantly affect the gain in the modulator of the standard HGHG setup and the first modulator in the OK-HGHG, since we operate at the lethargy regime. At the second modulator of the OK-HGHG setup, a higher current by itself would allow an increased gain in the second undulator. However, the higher energy spread counteracts this as seen in Eq. (2), and as a result, the ratios are only slightly increased compared to the nominal case. 
Finally, we studied a setup with increased modulator length by $20 \%$. This means that the periods of the modulator of the standard HGHG scheme, and of both modulators of the OK-HGHG scheme increased from 30 to 36 . This roughly corresponds to an increase of the length of each modulator from $2.48 \mathrm{~m}$ to $2.97 \mathrm{~m}$ and it directly increases the number of available power gain lengths from $L_{\text {mod }} / L_{\mathrm{g}}=2.6$ to $L_{\text {mod }} / L_{\mathrm{g}}=3.2$. For the standard seeding scheme, the energy modulation is increased proportionally with the length of the modulator in a first approximation [59]. With the OK-HGHG, the energy modulation in the first modulator is also increased proportionally to the modulator. However, in this case, we have the additional advantage that the gain is also increased in the second modulator. This led to a power ratio between 1300 and 900 for harmonics between the 8th and 15th as shown in Fig. 4(b), which is significantly increased compared to the

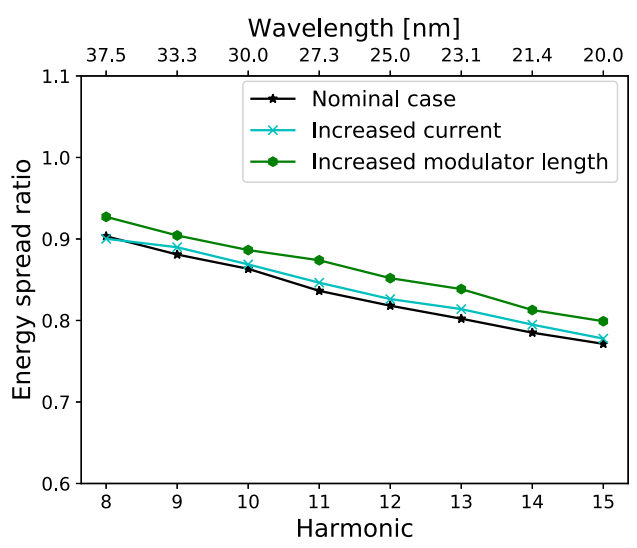

(a)

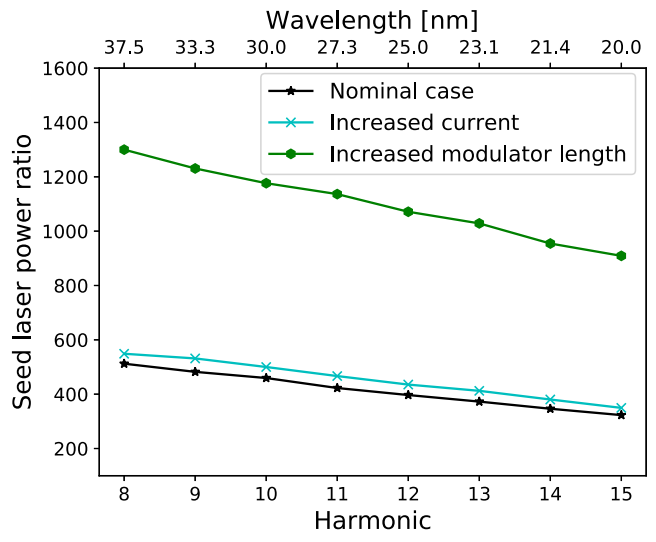

(b)

FIG. 4. (a) Required energy spread ratio between the two schemes $\left(\sigma_{\mathrm{E}, \mathrm{HGHG}}^{\prime} / \sigma_{\mathrm{E}, \mathrm{OK}-\mathrm{HGHG}}^{\prime}\right)$ to obtain $8 \%$ bunching at different harmonics. In addition to the standard parameters (Table I) also higher peak current and longer undulators are studied. The energy spread $\left(\sigma_{\mathrm{E}}^{\prime}\right)$ is measured upstream from the amplifier. (b) Ratio between the minimum seed laser power required for both schemes $\left(P_{\text {seed,HGHG }} / P_{\text {seed,OK-HGHG }}\right)$ to obtain the same bunching for the cases studied in (a). previously studied cases. It is worth noticing the essential dependence of the OK setup on the gain lengths. In order for this setup to be beneficial, at least 1.5 to 2 gain lengths are necessary for the second modulator, and the benefits increase strongly when increasing the gain lengths. From Eq. (2) and since $A \propto \sqrt{P_{\text {seed }}}$ [49], the gain in power increases with the square of the undulator periods at low gain. In this last setup studied, the increase of the undulator periods by $25 \%$, more than doubled the gain in power, which implies that there is exponential gain. A few more considerations about the power growth in the second modulator can be found in Ref. [47].

\section{B. Stability to fluctuations}

A significant advantage of seeded FELs is that they generate FEL pulses of great stability which lead to shot-toshot reproducibility. This is because seeding is a deterministic process, and the large signal to noise ratio eliminates the SASE fluctuations. However, there are still input parameters that fluctuate and that affect the bunching and hence the stability of the output FEL at the amplifier. Here, we investigate the effect of the seed laser power fluctuations and the electron beam compression factor fluctuations which changes both the peak current and the energy spread simultaneously. We compare the impact of these fluctuations on a standard HGHG and on an OKHGHG setup for the 10th harmonic of a $300 \mathrm{~nm}$ seed laser wavelength optimized to obtain $8 \%$ bunching, as described in this section. We show the results of this study in Fig. 5.

For the seed laser power fluctuations, once the laser power is optimized, both increasing and decreasing the power causes a decrease of the output bunching, as shown in Fig. 5(a). This bunching decrease is less significant for the OK-HGHG setup, because the energy modulation process only depends partly on the seed laser power which is important for the first modulator. The increase of the amplitude of the energy modulation in the second modulator does not depend anymore on the input seed laser power, and thus the bunching varies less than in a standard seeding HGHG setup. It should be noted that in both cases, a $10 \%$ variation of the seed laser power is affecting the bunching by less than $5 \%$ and $4 \%$ for the standard HGHG and the OK-HGHG setup, respectively.

For the compression factor fluctuations, we change proportionally the peak current and energy spread, while we keep all other parameters constant. In this case as well, the two stages of the OK-HGHG seem to be beneficial for the robustness of the setup. The increased energy spread is naturally affecting the bunching mechanism [see Eq. (1)] for both schemes, however, the increased peak current is only playing a role for the OK-HGHG. In the case of a standard HGHG setup, the modulator is operating at the lethargy regime where the peak current does not play any role on the energy modulation. Therefore, the bunching variation is dominated by the change of the energy spread. 


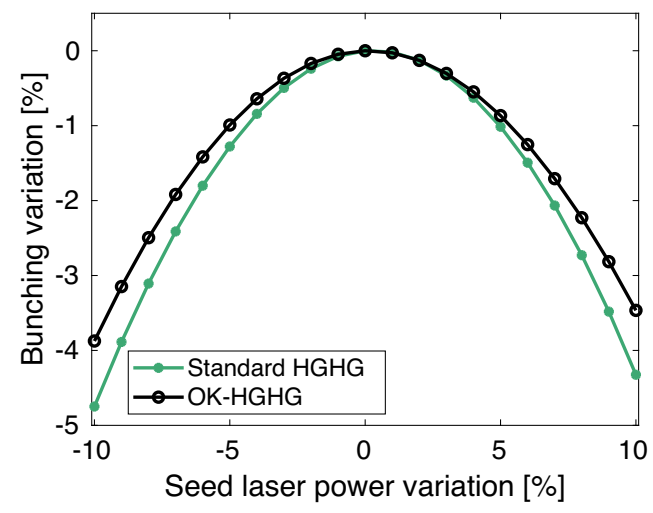

(a)

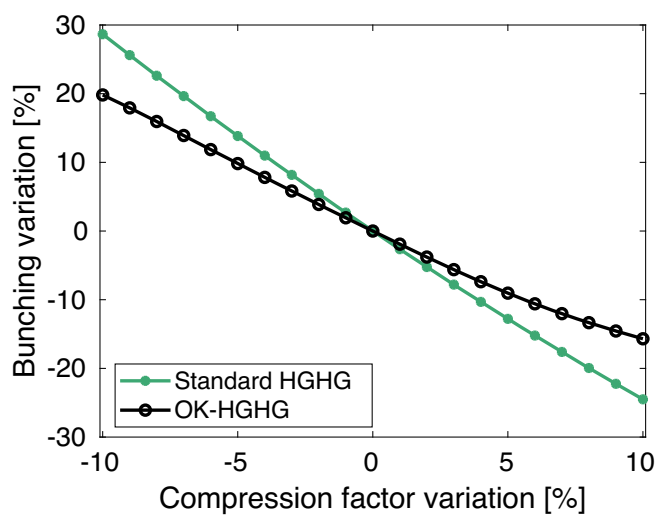

(b)

FIG. 5. (a) Bunching amplitude response to seed laser power fluctuations for a standard HGHG and an OK-HGHG setup. The nominal seed laser power is $27.7 \mathrm{MW}$ and $54.5 \mathrm{~kW}$ for the standard and the OK-HGHG, respectively. (b) Bunching amplitude response to electron beam compression factor fluctuations for a standard HGHG and an OK-HGHG setup. The nominal energy spread and peak current are shown in Table I. They change proportionally when we vary the compression factor.

Instead, the two stages of the optical klystron setup have different responses to the electron beam compression factor fluctuations and thus, its effect on the bunching is milder.

\section{FEL PERFORMANCE WITH AN OPTICAL KLYSTRON}

\section{A. Output FEL}

In this section, we show the FEL performance of the OKHGHG setup in comparison with a standard HGHG setup. We study with three dimensional and time-dependent simulations the performance of one of the most challenging cases; the 15th harmonic of a $300 \mathrm{~nm}$ seed laser wavelength, with an output wavelength of $20 \mathrm{~nm}$. At this high harmonic, for an HGHG setup, there are two concerns: (a) the energy spread upstream from the amplifier is relatively high to obtain sufficient bunching and (b) the signal to noise ratio is increased due to the frequency
TABLE I. Simulation parameters of the nominal case and for the harmonics between the 8th and 15th harmonic of a $300 \mathrm{~nm}$ seed laser wavelength. The seed laser peak power range refers to harmonics between the 8 th and 15 th. In some simulations, the temporal dependence is not used.

\begin{tabular}{lc}
\hline Electron beam & \\
\hline Energy $(E)$ & $750 \mathrm{MeV}$ \\
Uncorrelated energy spread $\left(\sigma_{E}\right)$ & $75 \mathrm{keV}$ \\
Current flattop $(I)$ & $500 \mathrm{~A}$ \\
Normalized emittance $\left(\epsilon_{x} / \epsilon_{y}\right)$ & $0.6 \mathrm{~mm} \mathrm{mrad}$ \\
Average beta functions $\left(\beta_{x} / \beta_{y}\right)$ & $8.4 \mathrm{~m} / 9.8 \mathrm{~m}$ \\
Bunch length (FWHM) & $110 \mathrm{fs}$ \\
\hline Input seed laser & $300 \mathrm{~nm}$ \\
\hline Wavelength $\left(\lambda_{\text {seed }}\right)$ & $33 \mathrm{fs}$ \\
rms duration & $42-265 \mathrm{~kW}$ \\
Peak power range $(\mathrm{OK}-\mathrm{HGHG})$ & $21-85 \mathrm{MW}$ \\
Peak power range (standard HGHG) & $757 \mu \mathrm{m}$ \\
Waist size & 5.42 \\
\hline Modulator & 30 \\
\hline Undulator parameter & $82.6 \mathrm{~mm}$ \\
\hline Number of periods $(N)$ & 74 \\
Undulator period & $33 \mathrm{~mm}$ \\
\hline Amplifier & 4 \\
\hline Periods per segment & \\
Undulator period & \\
Number of segments & \\
\hline \hline
\end{tabular}

multiplication [60]. The shot noise is expected to contribute quadratically with the harmonic number [61]. Nevertheless, we verified that for our set of parameters the 15 th harmonic is still possible and we present here the results.

For both cases, we start with $8 \%$ bunching upstream from the amplifier obtained with the minimum possible input seed laser power and we optimize the output FEL separately, in order to get maximum power and good spectral and temporal profile at the end of the amplifier. For both schemes, we follow the same procedure and we optimize the output FEL by fine-tuning the input seed laser power and the undulator parameter of the amplifier, $K_{\mathrm{amp}}$. With this optimization, we keep all amplifier segments tuned at the same resonance and there is no tapering taking place. The simulation parameters used are shown in Table I, while the fine-tuning results in the optimized simulation parameters are shown in Table II. In both cases, the

TABLE II. We list the simulation parameters optimized again for the time-dependent simulations, in addition to the parameters shown in Table I.

\begin{tabular}{lcc}
\hline \hline & Standard HGHG & OK-HGHG \\
\hline$R_{56,1} / R_{56,2}$ & $-/ 38.74 \mu \mathrm{m}$ & $482 \mu \mathrm{m} / 32.8 \mu \mathrm{m}$ \\
$K_{\text {amp }}$ & 1.792 & 1.789 \\
Seed laser power $\left(P_{\text {seed }}\right)$ & $61 \mathrm{MW}$ & $0.17 \mathrm{MW}$ \\
\hline \hline
\end{tabular}




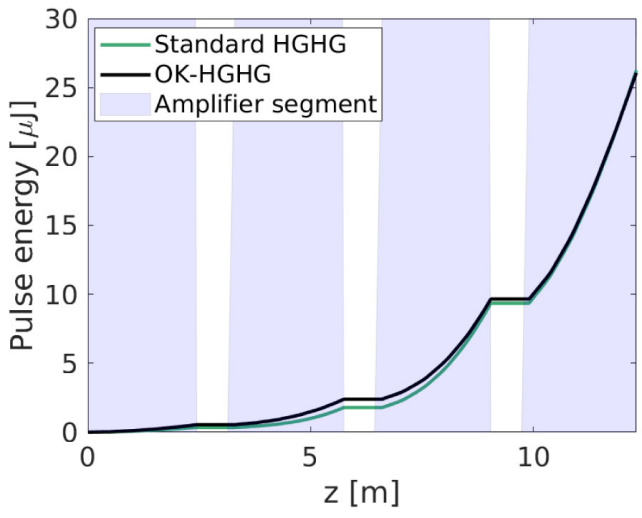

(a)

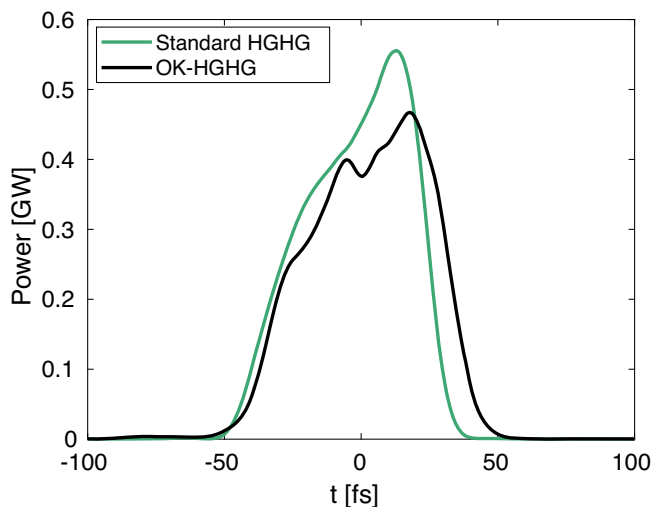

(b)

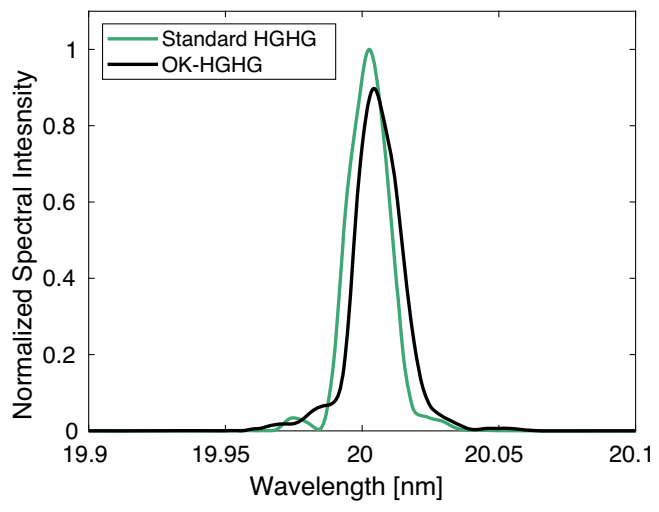

(c)

FIG. 6. We compare two fully optimized simulations of a standard HGHG and an OK-HGHG setup for the 15th harmonic of a $300 \mathrm{~nm}$ seed laser wavelength. (a) Gain curves in amplifier. (b) Output FEL power profiles. (c) Output FEL spectra normalized to the intensity of the standard HGHG simulation.

obtained bunching at the 15 th harmonic after optimization was roughly $7 \%$. Reducing the bunching from $8 \%$ to $7 \%$ was a result of the optimization and is the consequence of the high gain available within the used length of the amplifier.

The results of the optimization are shown in Fig. 6. The gain curve in the amplifier for both cases is comparable,
TABLE III. Comparison of simulation results between a standard HGHG and an OK-HGHG setup. The output wavelength is $20 \mathrm{~nm}$ and results as the 15 th harmonic of a $300 \mathrm{~nm}$ seed laser. The energy spread is calculated upstream from the amplifier.

\begin{tabular}{lcc}
\hline \hline & Standard HGHG & OK-HGHG \\
\hline Energy spread at amplifier $\left(\sigma_{E}^{\prime}\right)$ & $580.9 \mathrm{keV}$ & $766.4 \mathrm{keV}$ \\
FWHM relative bandwidth & $9 \times 10^{-4}$ & $9.3 \times 10^{-4}$ \\
Pulse energy & $26.3 \mu \mathrm{J}$ & $26 \mu \mathrm{J}$ \\
Pulse duration rms & $18.9 \mathrm{fs}$ & $21.5 \mathrm{fs}$ \\
Seed laser power $\left(P_{\text {seed }}\right)$ & $61 \mathrm{MW}$ & $0.17 \mathrm{MW}$ \\
\hline \hline
\end{tabular}

while the output power profile and spectra after $12.3 \mathrm{~m}$ in the amplifier are similar in terms of spectral intensity, bandwidth and power profile. We show in Table III an overview of the pulse properties of the output FEL. The slightly increased pulse duration with the OK-HGHG scheme is justified by the additional slippage in the second modulator.

\section{B. Shot-to-shot stability}

The use of a lower seed laser power in the OK-HGHG setup is expected to decrease the signal to noise ratio because of the shot noise that is unavoidably amplified as well in the amplifier $[52,60]$. In order to verify the effect of the lower signal to noise ratio, we simulated both schemes several times with different shot noises and we observed if the output FEL is sensitive to this change. As an example study, we use the simulations optimized and discussed above, shown in Fig. 6. We have randomly selected five cases with a different initial shot noise, since larger statistics are computationally time-demanding. For reference, we show in Fig. 7 the SASE background in the temporal domain for these five cases, using the OK-HGHG lattice and the same electron beam parameters, but without a seed laser.

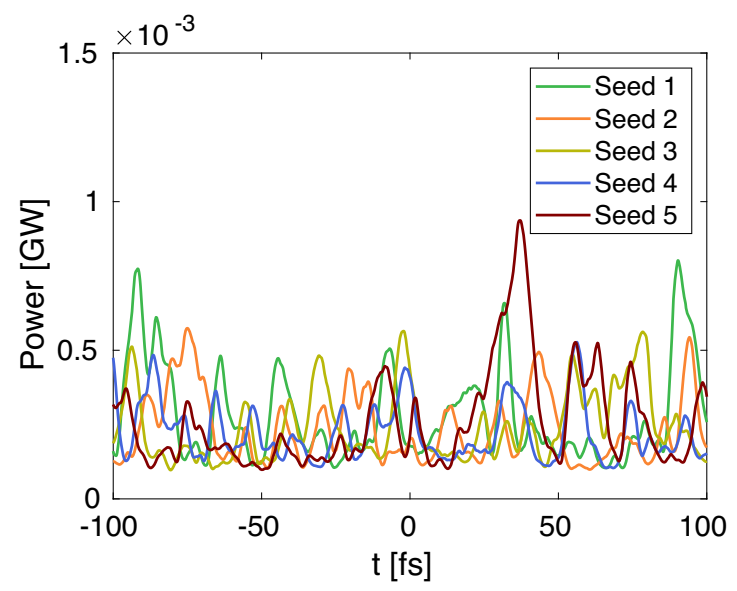

FIG. 7. SASE background in temporal domain for the five initial shot noise cases studied in this section. 


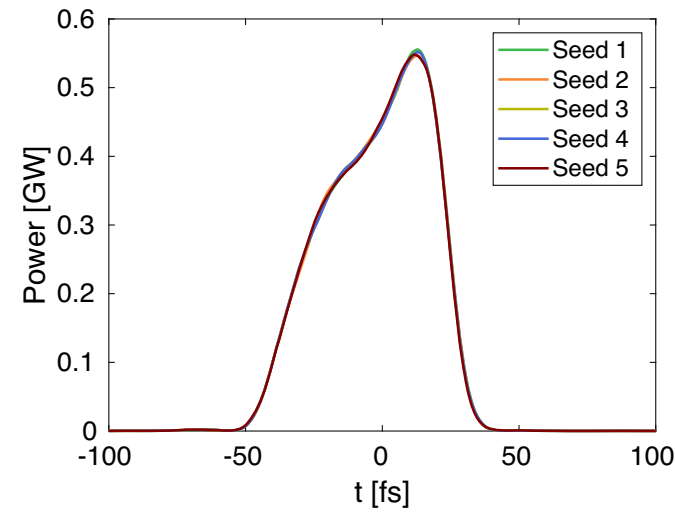

(a)

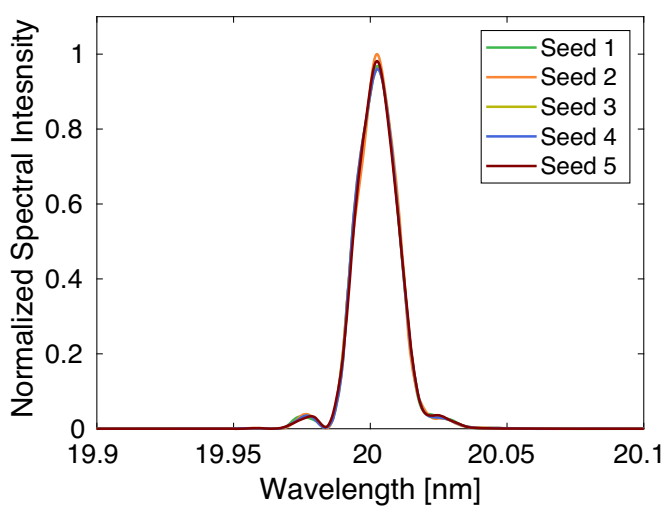

(b)

FIG. 8. Shot-to-shot fluctuations for a standard HGHG setup, imprinted on the output FEL at $20 \mathrm{~nm}$. (a) Power profile for different initial shot noise. (b) Spectra for different initial shot noise. They are normalized to the highest spectral intensity with Seed 2.

As expected, the performance of the standard HGHG is not affected at all by the initial shot noise, as it is shown in the spectrum and power profile in Fig. 8. On the contrary, in the OK-HGHG the output FEL properties have small variations on a shot-to-shot basis due to the sensitivity to shot noise, as shown in Fig. 9. Such variations, visible both in the temporal and spectral profiles, are very small in the case of an ideal electron beam as considered here and do not affect the possibility to implement seeding. The standard HGHG completely cancels the SASE background shown in Fig. 7, and the OK-HGHG almost completely cancels it too. The rms wavelength variation for the OKHGHG results reported in Fig. 9 is $0.001 \mathrm{~nm}$, and even though it is larger than the standard HGHG $(0.0001 \mathrm{~nm})$, it still remains significantly smaller than the FEL bandwidth $\left(\Delta \lambda_{\mathrm{rms}}=0.14 \mathrm{~nm}\right)$ and would not affect most experiments. Figure 10 shows that the overall energy per pulse can slightly fluctuate in an OK-HGHG as a result of the shot noise sensitivity, but also in this case the fluctuations are small. This limited sensitivity to shot noise might be partially compensated by the robustness of the OK-HGHG to

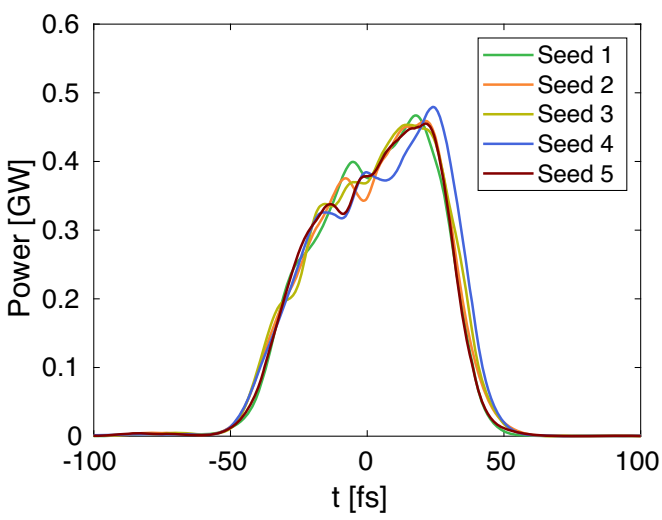

(a)

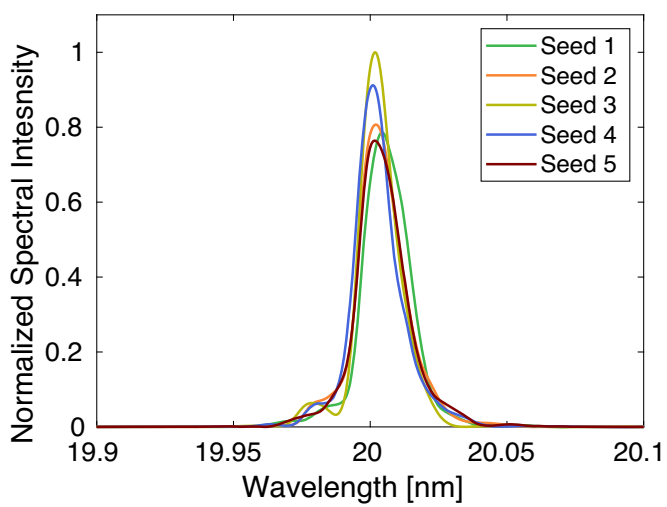

(b)

FIG. 9. Shot-to-shot fluctuations for an OK-HGHG setup, imprinted on the output FEL at $20 \mathrm{~nm}$. (a) Power profile for different initial shot noise. (b) Spectra for different initial shot noise. They are normalized to the highest spectral intensity with Seed 3.

other parameters' fluctuations as demonstrated in the previous section. All our studies suggest that the OKHGHG could reliably be used to extend the current capabilities of HGHG in the EUV spectral range.

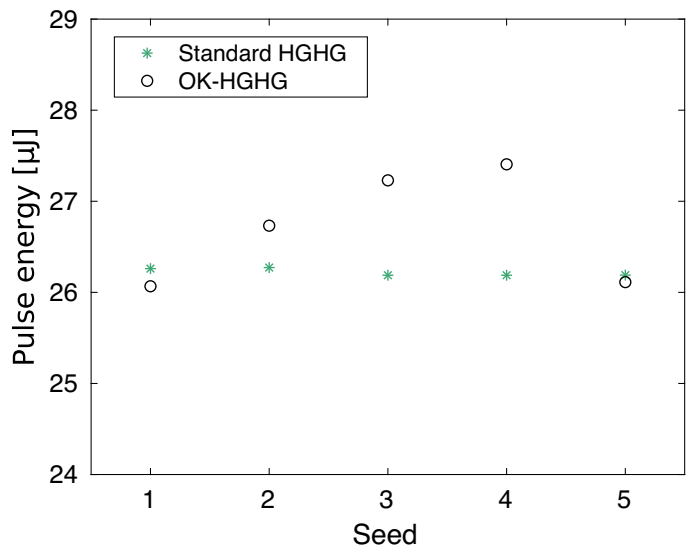

FIG. 10. Shot-to-shot fluctuations of pulse energy for the standard HGHG and the OK-HGHG setup. 


\section{OUTLOOK}

The need to increase the repetition rate of seeded radiation is becoming more and more important. Seeded radiation is nearly Fourier-limited and combines the stability with the control of spectral properties and timing. Being available at high repetition rate with no need for a monochromator will dramatically increase the control and statistics of experiments. Here, we considered an ideal electron beam distribution and we showed that an OKHGHG scheme can provide a significant reduction for the required seed laser power. This allows seed laser systems to immediately increase their repetition rate and allows the use of other types of short wavelength seed laser sources that are available only with lower peak powers. At the same time, the lower peak power allows operation with a larger laser transverse size which could reduce the sensitivity to the laser alignment. Since the OK-HGHG scheme does not require any additional components compared with an EEHG beamline, in many facilities it is possible to immediately test it and use it in a standard operation.

Complementary to Ref. [42] which presented very valuable experimental results for a single and two-stage HGHG, we have investigated the OK-HGHG setup in a systematic way with simulations. We have shown the strong dependence of the optical klystron on the gain lengths which can result in a reduction of the seed laser power by a factor of up to 1300 . We also showed that the two stages of the OK-HGHG scheme can provide increased stability to seed laser power and electron beam compression factor fluctuations. Most importantly, we have verified with time-dependent simulations that the low signal to noise ratio does not deteriorate the coherence properties of the output FEL from shot to shot.

As an outlook, the impact of electron beam imperfections should be studied to verify that they will not diminish its benefits, with a focus on electron beam energy chirp effects and the impact of microbunching which are known to be important for the operation of externally seeded FELs. In that direction, start-to-end simulations with a real electron beam of dedicated facilities would be valuable. Finally, the combination of the optical klystron with more complicated seeding schemes such as the EEHG and the cascaded HGHG should be systematically studied to achieve shorter output wavelengths.

\section{ACKNOWLEDGMENTS}

The authors would like to thank the Eugenio Ferrari for supporting the simulation work with Genesis 1.3, Sven Ackermann for a careful proofreading and Maxwell computational resources operated at Deutsches ElektronenSynchrotron (DESY), Hamburg, Germany. This work was supported by the Impuls- und Vernetzungsfond der Helmholtz-Gemeinschaft e.V. within the CAS-Helmholtz International Laboratory on Free-Electron Laser Science and Technology (CHILFEL), Grant No. InterLabs-0002.
[1] P. R. Ribic and G. Margaritondo, Status and prospects of $\mathrm{x}$-ray free-electron lasers (x-FELs): A simple presentation, J. Phy. D 45, 213001 (2012).

[2] J. Rossbach, J. R. Schneider, and W. Wurth, 10 years of pioneering $\mathrm{x}$-ray science at the free-electron laser flash at desy, Phys. Rep. 808, 1 (2019).

[3] B. Faatz et al., Simultaneous operation of two soft x-ray free-electron lasers driven by one linear accelerator, New J. Phys. 18, 062002 (2016).

[4] D. Nölle, FEL operation at the European XFEL facility, in Proceedings of International Free Electron Laser Conference (FEL'19), Hamburg, Germany, 2019 (JACoW, Geneva, Switzerland, 2019).

[5] T. Liu, X. Dong, and C. Feng, Start-to-end simulations of the reflection hard X-ray self-aeeding at the SHINE project, in Proceedings FEL'19, number 39 in Free Electron Laser Conference (JACoW, Geneva, Switzerland, 2019), pp. 254-257.

[6] E. Hemsing et al., Soft x-ray seeding studies for the SLAC Linac Coherent Light Source II, Phys. Rev. Accel. Beams 22, 110701 (2019).

[7] P. Gandhi, G. Penn, M. Reinsch, J. S. Wurtele, and W. M. Fawley, Oscillator seeding of a high gain harmonic generation free electron laser in a radiator-first configuration, Phys. Rev. ST Accel. Beams 16, 020703 (2013).

[8] V. Petrillo, M. Opromolla, A. Bacci, F. Broggi, I. Drebot, G. Ghiringhelli, E. Puppin, M. R. Conti, A. R. Rossi, M. Ruijter, S. Samsam, A. Tagliaferri, G. Rossi, and L. Serafini, Coherent, high repetition rate tender x-ray freeelectron laser seeded by an extreme ultra-violet freeelectron laser oscillator, New J. Phys. 22, 073058 (2020).

[9] N. S. Mirian, M. Opromolla, G. Rossi, L. Serafini, and V. Petrillo, High-repetition rate and coherent free-electron laser in the tender $\mathrm{x}$ rays based on the echo-enabled harmonic generation of an ultraviolet oscillator pulse, Phys. Rev. Accel. Beams 24, 050702 (2021).

[10] G. Paraskaki, S. Ackermann, B. Faatz, G. Geloni, T. Lang, F. Pannek, L. Schaper, and J. Zemella, Advanced scheme to generate $\mathrm{MHz}$, fully coherent FEL pulses at nm wavelength, Appl. Sci. 11, 6058 (2021).

[11] Z. Huang and R. D. Ruth, Fully Coherent X-ray Pulses from a Regenerative-amplifier Free-electron Laser, Phys. Rev. Lett. 96, 144801 (2006).

[12] K.-J. Kim and Y. V. Shvyd'ko, Tunable optical cavity for an X-ray free-electron-laser oscillator, Phys. Rev. ST Accel. Beams 12, 030703 (2009).

[13] K. Li and H. Deng, Systematic design and threedimensional simulation of $\mathrm{x}$-ray fel oscillator for shanghai coherent light facility, Nucl. Instrum. Methods Phys. Res., Sect. A 895, 40 (2018).

[14] G. Marcus et al., Regenerative amplification for a hard x-ray Free-Electron Laser, in Proceedings of FEL'19, number 39 in Free Electron Laser Conference (JACoW Publishing, Geneva, Switzerland, 2019), pp. 118-121.

[15] H. P. Freund, P. J. M. van der Slot, and Y. Shvyd'ko, An $\mathrm{x}$-ray regenerative amplifier free-electron laser using diamond pinhole mirrors, New J. Phys. 21, 093028 (2019).

[16] M. Opromolla, A. Bacci, M. R. Conti, A. R. Rossi, G. Rossi, L. Serafini, A. Tagliaferri, and V. Petrillo, High repetition rate and coherent free-electron laser oscillator in 
the tender x-ray range tailored for linear spectroscopy, Appl. Sci. 11, 5892 (2021).

[17] E. L. Saldin, E. A. Schneidmiller, and M. V. Yurkov, The Physics of Free Electron Lasers (Springer-Verlag, Berlin Heidelber, 2000).

[18] T. Ishikawa et al., A compact x-ray free-electron laser emitting in the sub-ångström region, Nat. Photonics 6, 540 (2012).

[19] P. Emma et al., First lasing and operation of an ångstromwavelength free-electron laser, Nat. Photonics 4, 641 (2010).

[20] H.-S. Kang et al., Hard X-ray free-electron laser with femtosecond-scale timing jitter, Nat. Photonics 11, 708 (2017).

[21] E. Prat et al., A compact and cost-effective hard x-ray freeelectron laser driven by a high-brightness and low-energy electron beam, Nat. Photonics 14, 748 (2020).

[22] J. Feldhaus, E. L. Saldin, J. R. Schneider, E. A. Schneidmiller, and M. V. Yurkov, Possible application of x-ray optical elements for reducing the spectral bandwidth of an x-ray SASE FEL, Opt. Commun. 140, 341 (1997).

[23] G. Geloni, V. Kocharyan, and E. Saldin, A novel selfseeding scheme for hard X-ray FELs, J. Mod. Opt. 58, 1391 (2011).

[24] J. Amann et al., Demonstration of self-seeding in a hardX-ray free-electron laser, Nat. Photonics 6, 693 (2012).

[25] L. H. Yu, Generation of intense UV radiation by subharmonically seeded single-pass free-electron lasers, Phys. Rev. A 44, 5178 (1991).

[26] G. Stupakov, Using the Beam-Echo Effect for Generation of Short-Wavelength Radiation, Phys. Rev. Lett. 102, 074801 (2009).

[27] D. Xiang and G. Stupakov, Echo-enabled harmonic generation free electron laser, Phys. Rev. ST Accel. Beams 12, 030702 (2009).

[28] S. Liu, W. Decking, V. Kocharyan, E. Saldin, S. Serkez, R. Shayduk, H. Sinn, and G. Geloni, Preparing for highrepetition rate hard X-ray self-seeding at the European X-ray Free Electron Laser: Challenges and opportunities, Phys. Rev. Accel. Beams 22, 060704 (2019).

[29] C. Feng et al., Coherent extreme ultraviolet free-electron laser with echo-enabled harmonic generation, Phys. Rev. Accel. Beams 22, 050703 (2019).

[30] P. Ribič et al., Coherent soft x-ray pulses from an echoenabled harmonic generation free-electron laser, Nat. Photonics 13, 555 (2019).

[31] E. Allaria et al., Highly coherent and stable pulses from the FERMI seeded free-electron laser in the extreme ultraviolet, Nat. Photonics 6, 699 (2012).

[32] K. Prince et al., Coherent control with a short-wavelength free electron laser, Nat. Photonics 10, 176 (2016).

[33] P. K. Maroju et al., Attosecond pulse shaping using a seeded free-electron laser, Nature (London) 578, 386 (2020).

[34] A. Wituschek et al., Tracking attosecond electronic coherences using phase-manipulated extreme ultraviolet pulses, Nat. Commun. 11, 883 (2020).

[35] C. Lechner et al., Seeding R\&D at sFLASH, in Proceedings of FEL'19, number 39 in Free Electron Laser
Conference (JACoW Publishing, Geneva, Switzerland, 2019), pp. 230-233.

[36] C. Heyl, C. Arnold, A. Couairon, and A. L'Huillier, Introduction to macroscopic power scaling principles for high-order harmonic generation, J. Phys. B 50, 013001 (2017).

[37] G. Lambert et al., Injection of harmonics generated in gas in a free-electron laser providing intense and coherent extreme-ultraviolet light, Nat. Phys. 4, 296 (2008).

[38] S. Ackermann et al., Generation of Coherent 19- and 38-nm Radiation at a Free-Electron Laser Directly Seeded at $38 \mathrm{~nm}$, Phys. Rev. Lett. 111, 114801 (2013).

[39] M. Labat et al., High-Gain Harmonic-generation freeelectron laser seeded by harmonics generated in gas, Phys. Rev. Lett. 107, 224801 (2011).

[40] L. Giannessi et al., Superradiant Cascade in a Seeded FreeElectron Laser, Phys. Rev. Lett. 110, 044801 (2013).

[41] N. A. Vinokurov and A. N. Skrinsky, About the maximum power of an optical klystron on a storage ring, Preprint of INP 77-59, pp. 77-69, 1977.

[42] J. Yan et al., Self-amplification of Coherent Energy Modulation in Seeded Free-Electron Lasers, Phys. Rev. Lett. 126, 084801 (2021).

[43] G. Paraskaki, E. Allaria, M. Yurkov, J. Zemella, and E. Schneidmiller, Options for high-repetition-rate seeded FEL, in EUV and X-ray Optics, Sources, and Instrumentation, edited by R. Hudec, L. Pina, T. Tschentscher, L. Juha, L. Patthey, K. Tiedtke, M. Zangrando, S. Bajt, and S. Guizard (International Society for Optics and Photonics, SPIE, Czech Republic, 2021), Vol. 11776, pp. 90-99, 10.1117/12.2589693.

[44] E. Allaria et al., FLASH2020+ plans for a new coherent source at DESY, in Proceedings of 10th International Particle Accelerator Conference (IPAC'21) (JACoW Publishing, Geneva, 2021).

[45] J. Yan, N. Huang, H. Deng, B. Liu, D. Wang, and Z. Zhao, First observation of laser-beam interaction in a dipole magnet, Adv. Opt. Photonics 3, 045003 (2021).

[46] X. Wang, C. Feng, B. Faatz, W. Zhang, and Z. Zhao, Direct-amplification enabled harmonic generation for seeding a high-repetition-rate free-electron laser, https://arxiv .org/abs/2103.11971.

[47] Q. Jia, Analysis of modulation parameters for high repetition rate seeded FEL, Nucl. Instrum. Methods Phys. Res., Sect. A 1015, 165767 (2021).

[48] G. Penco, G. Perosa, E. Allaria, S. Di Mitri, E. Ferrari, L. Giannessi, S. Spampinati, C. Spezzani, and M. Veronese, Enhanced seeded free electron laser performance with a "cold" electron beam, Phys. Rev. Accel. Beams 23, 120704 (2020).

[49] L. H. Yu and J. Wu, Theory of high gain harmonic generation: An analytical estimate, Nucl. Instrum. Methods Phys. Res., Sect. A 483, 493 (2002).

[50] G. Dattoli, L. Giannessi, and P. L. Ottaviani, MOPA optical klystron FELs and coherent harmonic generation, Nucl. Instrum. Methods Phys. Res., Sect. A 507, 26 (2003).

[51] Y. Ding, P. Emma, Z. Huang, and V. Kumar, Optical klystron enhancement to self-amplified spontaneous emission free electron lasers, Phys. Rev. ST Accel. Beams 9, 070702 (2006). 
[52] G. Penco, E. Allaria, G. De Ninno, E. Ferrari, and L. Giannessi, Experimental Demonstration of Enhanced SelfAmplified Spontaneous Emission by an Optical Klystron, Phys. Rev. Lett. 114, 013901 (2015).

[53] G. Penco, E. Allaria, G. De Ninno, E. Ferrari, L. Giannessi, E. Roussel, and S. Spampinati, Optical Klystron Enhancement to Self Amplified Spontaneous Emission at FERMI, Photonics 4, 15 (2017).

[54] E. L. Saldin, E. A. Schneidmiller, and M. V. Yurkov, The free electron laser klystron amplifier concept, in Proceedings of 26th International Free Electron Laser Conference \& 11th FEL Users Workshop (FEL'04)(JACoW Publishing, Geneva, 2004).

[55] Y. Ding, P. Emma, Z. Huang, and V. Kumar, Optical klystron enhancement to self-amplified spontaneous emission free electron lasers, Phys. Rev. Accel. Beams 9, 070702 (2006); Phys. Rev. Accel. Beams 23, 019901(E) (2020).

[56] G. Geloni, M. Guetg, S. Serkez, and E. Schneidmiller, Revision of optical klystron enhancement effects in self-amplified spontaneous emission free electron lasers, Phys. Rev. Accel. Beams 24, 090702 (2021).

[57] M. Beye, FLASH2020+: Making FLASH Brighter, Faster and More Flexible: Conceptual Design Report (Verlag Deutsches Elektronen-Synchrotron, Hamburg, 2020).

[58] S. Reiche, Genesis 1.3: A fully 3d time-dependent fel simulation code, Nucl. Instrum. Methods Phys. Res., Sect. A 429, 243 (1999).

[59] E. Hemsing, G. Stupakov, D. Xiang, and A. Zholents, Beam by design: Laser manipulation of electrons in modern accelerators, Rev. Mod. Phys. 86, 897 (2014).

[60] E. L. Saldin, E. A. Schneidmiller, and M. V. Yurkov, Study of a noise degradation of amplification process in a multistage hghg fel, Opt. Commun. 202, 169 (2002).

[61] W. Brefeld et al., Study of the frequency multiplication process in a multistage HGHG FEL, Nucl. Instrum. Methods Phys. Res., Sect. A 483, 80 (2002). 